\title{
A Class of Modular Aerial Robots
}

\author{
Roberto Naldi, Francesco Forte and Lorenzo Marconi
}

CASY-DEIS, Università di Bologna, Bologna, 40133, Italy.

\begin{abstract}
In this work we consider the modelling of a modular aerial robot obtained by rigidly interconnecting a certain number of ducted-fan aircraft. It is shown how different geometric configurations lead to different dynamical properties of the overall system which can be exploited to improve the achievable closed-loop performances in certain scenarios. Suitable constrained control allocation problems are formulated to show how the modular system performs in presence of possible extra payload and of force and torque disturbances that may derive from the physical interaction with the surrounding environment.
\end{abstract}

\section{INTRODUCTION}

Recent applications of aerial robotics have shown the capability of miniature unmanned vehicles to accomplish tasks that require a physical interaction between the vehicle and the surrounding environment. These kind of operations, which include cooperative grasping and transportation [11], cleaning [2], docking [10], and others, represent a new research direction for both the design and control of aerial vehicles. The vision, which inspired also the European Project AIRobots [1], is to allow airborne systems to succeed in many service robotics operations [14] that are usually achieved only by robots fixed on the ground.

In this work we address the modelling of a new class of aerial service robots based on the ducted-fan configuration [9]. Ducted-fan aerial vehicles, having the propeller protected by a shroud, are in fact capable to physically interact with the surrounding environment safely and, for this reason, they represent a natural aero-mechanical choice for the envisioned application scenario. This configuration, however, is characterized by some relevant payload and dynamical constraints that may reduce its effectiveness in certain applications. First of all, as shown, for instance, in [12], the position of the payload, and thus the position of the center of mass, play an important role in determining the stability of the given configuration since the torques required to govern the attitude dynamics are produced by applying aerodynamic forces at a given distance from the center of mass itself. Moreover, because of under-actuation, the number of degrees of freedom that can be actually controlled independently may not be enough in certain situations in which the robot has to operate (such has narrow environments).

This research is framed within the collaborative project AIRobots (Innovative Aerial Service Robots for Remote inspections by contact, ICT 248669) supported by the European Community under the 7th Framework Programme. Corresponding author: Roberto Naldi, email: roberto.naldi@unibo.it
To overcome these limitations, the idea pursued in this paper is to focus on a modular structure that may be expanded by rigidly interconnecting two or more basic airframe module (i.e. ducted-fan airframes).

Modularity has several advantages, and in particular it allows one to overcome the limitations of each single module without re-designing a different vehicle. Goal of this work is to start investigating how the actuator redundancy, characterizing a modular vehicle, can be combined with the geometric properties of the formation to obtain certain desired dynamical properties, such as robustness to force / torque external disturbances and payload variations. To this purpose, we analyze the feasibility of some control allocation problems (see, among others, [3], [6] and the references therein).

The idea of aerial systems composed of different modules is not new in literature. In this respect, it is worth mentioning the experiments proposed in [13], in which the aerial vehicle is obtained by autonomously merging a number of very simple airframe modules, and the cooperative transportation proposed in [11], in which several quad-rotors are employed to transport a certain payload forming a rigid modular system. With respect to the above works, the emphasis here is to characterize the dynamical properties of the overall system as functions of the geometric characteristics of the final modular system.

The paper is organized as follows. Section II derives the dynamical model of a general ducted-fan modular system. Section III proposes the control allocation problems that are used to analyze the dynamical properties of the different configurations. The latter are presented in Sections IV and $\mathrm{V}$, by also showing the numerical results obtained for a real physical prototype. Finally Section VI presents final remarks.

\section{The Modular Aerial Vehicle}

\section{A. Single Module}

Forces and torques components generated by each $i-t h$ single module are expressed in the reference body frames $F_{b_{i}}=\left\{O_{b_{i}}, \vec{i}_{b_{i}}, \vec{j}_{b_{i}}, \vec{k}_{b_{i}}\right\}$ attached to the center of mass of each module. As shown in Figure 1, each body reference frame is fixed with the $z$-axis aligned with the propeller spin axis.

According to [12], each ducted-fan aerial vehicle can be thought as composed by two main subsystems. The first one consists of a fixed-pitch propeller driven by an electric motor. This subsystem has the fundamental role of generating the 
main thrust $T$ required to counteract the gravity force by inducing an airflow inside the duct. The speed vector of the induced airflow is assumed to be aligned with the body $z$ axis. The second subsystem consists of a set of control vanes which are positioned below the propeller. The vanes are immersed into the airflow induced by the propeller and their angular positions are controlled to generate a certain number of aerodynamic lift and drag forces. Each lift force $L$, see [15], is perpendicular to the airflow speed vector and its magnitude, for small angle of attack values, is given by

$$
L=\frac{1}{2} \rho c_{L} \alpha S V^{2}
$$

in which $\rho$ is the air density, $c_{L}$ the lift coefficient, which depends on the specific airfoil characteristics, $S$ the airfoil's surface, $V$ the speed of the airflow and $\alpha$ the angle of attack of the vane. The drag forces, by definition, are directed along the airflow speed vector (i.e. along the body $z$-axis) and, since their magnitude is small for reasonably small angles of attack, they are assumed to be compensated by the propeller thrust $T$ and then neglected in the analysis carried out in this work.

For the ducted-fan configuration, the vanes can be divided into three groups (see [12]). The first two groups are designed to generate respectively a resultant force contribution $F_{x}$ and $F_{y}$ directed along the body $x$ and $y$ axis. The third group, using the fact that the lift of two opposite vanes may produce a pair of antagonist forces, is able to produce a resultant torque component $\tau_{z}$ along the body $z$ axis, and thus to compensate for the propeller torque disturbances. In particular, recalling (1) and since, according to Froude's momentum theory (see [16]), the magnitude of the induced airflow $V$ is proportional to $\sqrt{T}$, we have

$$
\begin{aligned}
& F_{x}\left(\alpha_{x}\right):=k_{L} \alpha_{x} T \quad F_{y}\left(\alpha_{y}\right):=k_{L} \alpha_{y} T \\
& \tau_{z}\left(\alpha_{z}\right):=k_{L}^{\prime} \alpha_{z} T
\end{aligned}
$$

where $k_{L}$ and $k_{L}^{\prime}$ are constant coefficients collecting all the aerodynamic parameters, while $\alpha_{x}, \alpha_{y}$ and $\alpha_{z}$ are the angles of attack of the control vanes in each one of the three above different groups.

From the above arguments the force-torque (wrench) vector produced by each ducted-fan module is given by

$$
w:=K(T) u
$$

in which $u:=\left[\begin{array}{cccc}T & \alpha_{x} & \alpha_{y} & \alpha_{z}\end{array}\right]^{T}$ and $K(T):=$ $\operatorname{diag}\left(1, k_{L} T, k_{L} T, k_{L}^{\prime} T\right)$. Let $d$ be the distance, along the body $z$ axis, between the center of pressure of the aerodynamic forces and the center of mass of the vehicle. Accordingly, forces $F_{x}$ and $F_{y}$ produce also two torque components around the $x$ and $y$ axis of the rigid body. This fact is exploited in the following section where the resultant control wrench vector of a generic modular system, including also the single module case, is derived.

In the following we assume that each module operates near the hovering condition, namely, the input $T$ is close to the value $T_{\text {hov }}=M_{m} / N>0$ depending on the overall weight of the modular system $M_{m}$ and the number $N$ of equal modules which are interconnected to form the final desired modular unit. Under the above condition, the matrix $K(T)$ relating force-torque vector $w$ and the input $u$ can be assumed constant and equal to $K(T) \approx K\left(T_{\text {hov }}\right)$.

We will assume that the input $T$ belongs to the set $\Omega_{\bar{T}}:=$ $\left\{T \in \mathbb{R}:\left|T-T_{\text {hov }}\right| \leq \bar{T}\right\}$, for some positive $\bar{T}<<T_{\text {hov }}$, and that, due to aerodynamical limitations, each angle of attack $\alpha_{j}, j \in\{x, y, z\}$, belong to the set $\Omega_{\bar{\alpha}}:=\{\alpha \in \mathbb{R}$ : $|\alpha| \leq \bar{\alpha}\}$. Accordingly, we have

$$
w \in \Omega_{w}
$$

in which $\Omega_{w} \subset \mathbb{R}^{4}, i \in\{1,2, \ldots N\}$, denotes the image of the set $\Omega_{\bar{T}} \times \Omega_{\bar{\alpha}} \times \Omega_{\bar{\alpha}} \times \Omega_{\bar{\alpha}}$ through the linear map $K\left(T_{h o v}\right)$.

For further details about the modeling of this class of systems the reader is referred to [8] and [17] and reference therein.

\section{B. Modular Configuration}

Let us consider a modular system composed of a number $N>0$ of equal ducted-fan modules rigidly connected together as sketched, for instance, in Figures 2(a), 2(b), 1. Let the coordinate frame $F_{b_{m}}=\left\{O_{b_{m}}, \vec{i}_{b_{m}}, \vec{j}_{b_{m}}, \vec{k}_{b_{m}}\right\}$ be attached to the center of mass of the modular system. Let the unit vectors $\vec{k}_{b_{i}}$, namely the propellers spin axis of each module $i, i \in\{1,2, \ldots N\}$, be fixed in order to point in the same direction, so that the propeller thrust of each module counteracts the gravity force at hover. Finally, for all the vehicles in the group in which the body $z$-axis does not intersect the center of mass of the formation, let the $x$ axis be directed in a way that it intersects the $z$-axis of the reference frame $F_{b_{m}}$ as shown, for instance, in Figures 1 and 2(a), while, for the remaining modules, let the $x$-axis be aligned with the one of the frame $F_{b_{m}}$, as shown in Figure 2(b). For the above choice of reference frames, the vectors $\ell_{i}^{b_{i}}$, which denote the position of the center of mass of each module with respect to the center of mass of the overall formation expressed in the reference frame $F_{b_{i}}$, are given by

$$
\ell_{i}^{b_{i}}=\left[r_{i}, 0, h_{i}\right]^{T}
$$

in which, by construction, $r_{i} \in \mathbb{R}_{\geq 0}$ and $h_{i} \in \mathbb{R}$ denote respectively the horizontal and vertical displacement of each $\mathrm{UAV}$ in the group, with $i \in\{1,2, \ldots N\}$. Finally, let us denote the relative orientation between the reference frames $F_{b_{i}}$ and the reference frame $F_{b_{m}}$ by $\psi_{i}, i \in\{1,2, \ldots, N\}$. Indeed, $\psi_{i}$ denotes the angle by which the reference frame of each single module should be rotated around the $z$-axis in order to align the $x$-axis with the one of the frame $F_{b_{m}}$.

Since modules are physically separated, the distance between the center of mass of any two different modules is positive. Accordingly, if $N>1$, the following constraint on system parameters holds

$$
\left\|R_{\psi_{i}} \ell_{i}^{b_{i}}-R_{\psi_{j}} \ell_{j}^{b_{j}}\right\|>0 \text { for all } i \neq j, i, j \in\{1,2, \ldots, N\}
$$

where

$$
R_{\psi_{i}}:=\left[\begin{array}{ccc}
\cos \psi_{i} & -\sin \psi_{i} & 0 \\
\sin \psi_{i} & \cos \psi_{i} & 0 \\
0 & 0 & 1
\end{array}\right]
$$


With the above construction and notation at hand, we are now able to give a definition of the class of modular system of interest.

Definition 1 A modular ducted-fan aerial robot $\mathcal{M}$ is given by the 4-tuple $(N, \Psi, \mathcal{R}, \mathcal{H})$ where

- $N$ is the number of modules generating a force-torque vector $w \in \mathbb{R}^{4}$ given by (3) and satisfying (4);

- $\Psi:=\left\{\psi_{i} \in \mathbb{R} \mid i=1,2, \ldots N\right\}$ is the set of the orientations $\psi_{i}$ of each frames $F_{b_{i}}$ with respect to the frame $F_{m}$;

- $\mathcal{R}:=\left\{r_{i} \in \mathbb{R} \mid i=1,2, \ldots N\right\}$ is the set of horizontal displacement $r_{i}$ between each module and the center of gravity of the formation;

- $\mathcal{H}:=\left\{h_{i} \in \mathbb{R} \mid i=1,2, \ldots N\right\}$ is the set of vertical displacement $h_{i}$ between each module and the center of gravity of the formation.

Let $\bar{w}:=\left[w_{1}^{T}, w_{2}^{T}, \ldots, w_{N}^{T}\right]^{T}$ be the vector of all the force-torque components $w_{i}$ produced by all the $N$ modules in the formation. According to (4) we have

$$
\bar{w} \in \Omega_{\bar{w}}, \quad \Omega_{\bar{w}}:=\left\{\Omega_{w_{1}} \times \Omega_{w_{2}} \times \ldots \times \Omega_{w_{N}}\right\} \subset \mathbb{R}^{4 N} .
$$

The resultant control force and torque vectors $f_{c} \in \mathbb{R}^{3}$ and $\tau_{c} \in \mathbb{R}^{3}$ applied by all the modules to the center of mass of the formation are given by

$$
\begin{gathered}
f_{c}=B_{f}(\Psi) \bar{w} \\
\tau_{c}=B_{\tau}(\Psi, \mathcal{R}, \mathcal{H}) \bar{w}
\end{gathered}
$$

where

$$
\begin{aligned}
B_{f}(\Psi) & :=\left[R_{\psi_{1}} G_{f}, \ldots, R_{\psi_{N}} G_{f}\right] \\
B_{\tau}(\Psi, \mathcal{R}, \mathcal{H}) & :=\left[R_{\psi_{1}} G_{\tau}\left(r_{1}, h_{1}\right), \ldots, R_{\psi_{N}} G_{\tau}\left(r_{N}, h_{N}\right)\right],
\end{aligned}
$$

having defined

$$
G_{f}:=\left[\begin{array}{cccc}
0 & 1 & 0 & 0 \\
0 & 0 & 1 & 0 \\
-1 & 0 & 0 & 0
\end{array}\right],
$$

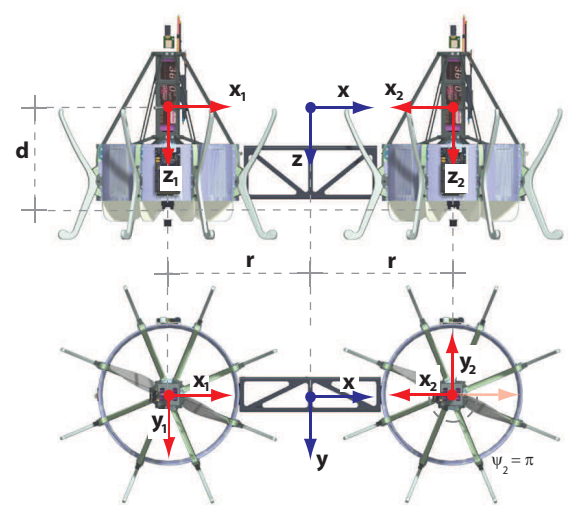

Fig. 1. A planar ducted-fan modular aerial robot with $N=2$.

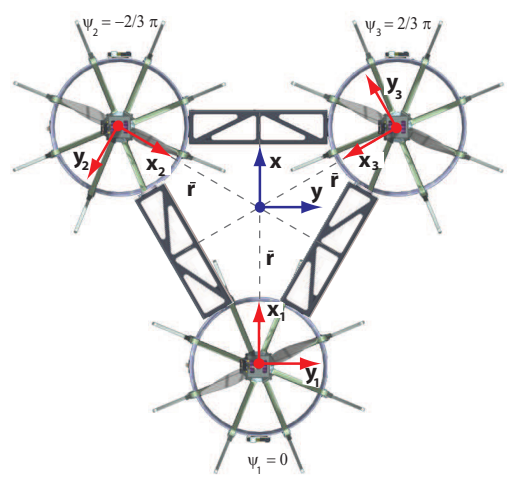

(a) $N=3$ planar design.

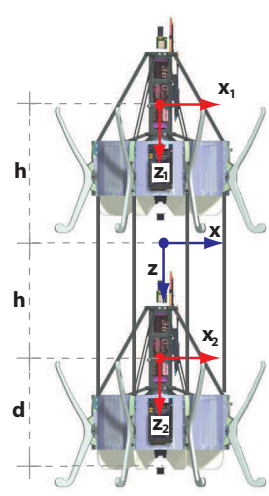

(b) $N=2$ FA design.
Fig. 2. A planar ducted-fan modular aerial robot with $N=3$ and a modular aerial robot with $N=2$ and modules aligned vertically.

\section{Vehicle Dynamics}

A mathematical model for the modular system can be derived using the Newton-Euler equations of motion of a rigid body in the configuration space $S E(3)=\mathbb{R}^{3} \times$ $S O(3)$. By considering the inertial coordinate frame $F_{i}=$ $\left\{O_{i}, \vec{i}_{i}, \vec{j}_{i}, \vec{k}_{i}\right\}$ and assuming that the body frame $F_{b_{m}}$ has its axis aligned with the principal axis of inertia of the rigid body, the dynamical model of the modular ducted-fan aerial robot $\mathcal{M}$ with respect to the inertial frame is described by ${ }^{1}$

$$
\begin{aligned}
M_{m} \ddot{p} & =R f_{c}+M_{m} g e_{3}+f_{d} \\
J_{m} \dot{\omega} & =-\omega \times J_{m} \omega+\tau_{c}+\tau_{d}
\end{aligned}
$$

where $M_{m}$ is the total mass of the system, $J_{m}$ denotes the inertia of the vehicle, $p=\operatorname{col}(x, y, z)$ is the position of the center of mass, $\omega$ the angular velocity expressed in the body frame $F_{b_{m}}, R$ the rotation matrix relating the reference frames $F_{b_{m}}$ and $F_{i}$, and $e_{3}$ the unit vector $e_{3}:=[0,0,1]^{T}$. Moreover $f_{d}$ and $\tau_{d}$ represent respectively a force and a torque disturbance applied to the vehicle, caused by the presence of additional payload or by external forces produced by the interaction with the environment.

\section{Control Allocation}

For systems characterized by actuators redundancy the design of the control law may be simplified by considering a control allocation problem ( [3], [6], [5]). In particular, for the modular ducted-fan aerial robot given in Definition 1, it is worth considering two different control allocation problems having as a final goal to assign different components of the wrench vector.

The first one, denoted as fully actuated control allocation problem (FA-CAP), consists of finding a choice of the vector of control inputs $\bar{w}$ in order to produce a final desired wrench vector $w_{c}^{\star} \in \mathbb{R}^{6}$. The idea is to obtain a fully-actuated vehicle in which all the 6 degrees of freedom of the rigid body dynamics can be controlled separately.

\footnotetext{
${ }^{1}$ We define $\omega \times:=\operatorname{Skew}(\omega)$ where $\operatorname{Skew}\left(\operatorname{col}\left(x_{1}, x_{2}, x_{3}\right)\right)$ denotes the skew-symmetric matrix with the first, second and third row respectively given by $\left[0,-x_{3}, x_{2}\right],\left[x_{3}, 0,-x_{1}\right]$ and $\left[-x_{2}, x_{1}, 0\right]$.
} 
The problem can be formulated as follows.

Problem FA-CAP: Given $w_{c}^{\star} \in \mathbb{R}^{6}$, find a value of $\bar{w} \in \mathbb{R}^{4 N}$ such that

$$
B_{F A}(\Psi, \mathcal{R}, \mathcal{H}) \bar{w}=w_{c}^{\star}
$$

having defined

$B_{F A}(\Psi, \mathcal{R}, \mathcal{H}):=\left[\begin{array}{cc}B_{f}(\Psi)^{T} & B_{\tau}(\Psi, \mathcal{R}, \mathcal{H})^{T}\end{array}\right]^{T}$.

In the second control allocation problem that is considered here, it is of interest to assign the torque vector and the force component directed along the body $z$-axis. The idea behind this problem is to govern the vehicle by using vectored-thrust control paradigms - see [7] - for a general VTOL aerial robot, without necessarily requiring the system to be fully actuated. The problem is referred to as vectored-thrust control allocation problem (VT-CAP) and it is formulated as follow.

Problem VT-CAP: Given $T_{c}^{\star} \in \mathbb{R}$ and $\tau_{c}^{\star} \in \mathbb{R}^{3}$, find a value of $\bar{w} \in \mathbb{R}^{4 N}$ such that

$$
B_{V T}(\Psi, \mathcal{R}, \mathcal{H}) \bar{w}=\left[\begin{array}{c}
T_{c}^{\star} \\
\tau_{c}^{\star}
\end{array}\right]
$$

in which $B_{V T}(\Psi, \mathcal{R}, \mathcal{H})$ is given by the last four rows of $B_{F A}(\Psi, \mathcal{R}, \mathcal{H})$.

From basic linear algebra, it follows that sufficient conditions for Problems FA-CAP and VT-CAP to be feasible are given respectively by

$$
\begin{aligned}
& \operatorname{rank}\left(B_{F A}(\Psi, \mathcal{R}, \mathcal{H})\right)=6 \\
& \operatorname{rank}\left(B_{V T}(\Psi, \mathcal{R}, \mathcal{H})\right)=4
\end{aligned}
$$

As shown in Section IV, conditions (12) and (13) can be more precisely characterized in term of the number of modules and the geometric properties employed to build the modular aerial robot.

In order to design control allocation policies actually employable on a real physical system, it is also necessary to take into account the constraint (6) on the control input vector $\bar{w}$. The problems FA-CAP and VT-CAP, with the additional requirement that the vector $\bar{w}$ solution to (10) and, respectively, to (11) fulfills also (6), are denoted by FA-CCAP and VT-CCAP, respectively.

In order to check the feasibility of the above constrained control allocation problems, it is of interest to study the set of attainable forces and moments under any choice of $\bar{w} \in \Omega_{\bar{\omega}}$. In particular we will consider the two compacts sets

$$
\begin{aligned}
& \Phi_{f}:=\left\{f \in \mathbb{R}^{3}: f=B_{f}(\Psi) \bar{w}, \bar{w} \in \Omega_{\bar{w}}\right\} \\
& \Phi_{\tau}:=\left\{\tau \in \mathbb{R}^{3}: \tau=B_{\tau}(\Psi, \mathcal{R}, \mathcal{H}) \bar{w}, \bar{w} \in \Omega_{\bar{w}}\right\} .
\end{aligned}
$$

The sets $\Phi_{f}$ and $\Phi_{\tau}$ are employed to characterize some fundamental control properties attainable for the closed loop system. This fact will be investigated in details in Section V.

\section{Geometric Properties}

The feasibility of Problems FA-CAP and VT-CAP, characterized by conditions (12) and (13), strongly depends on the given number of interconnected modules and on the characteristics of the adopted geometric configuration for the modular aerial robot. Interestingly enough, the geometric conditions (12) and (13) also depend on the presence or not of possible payload attached to the formation. To this end, in order to more precisely characterize the geometric constraints of the modular aerial robot, we consider first the following assumption.

Assumption 1: the position of the center of mass of the interconnected system is given by the centroid formed by all the centers of mass of each single module, namely

$$
\sum_{i=1}^{N} R_{\psi_{i}} \ell_{i}^{b_{i}}=\left[\begin{array}{lll}
0 & 0 & 0
\end{array}\right]^{T} .
$$

Assumption 1 clearly holds for the special case in which no additional payload other than the modules is carried by the formation. In this special condition it is possible to characterize the minimum number of modules required to satisfy conditions (12) and (13).

Proposition 1 Consider a modular system $\mathcal{M}=$ $(N, \Psi, \mathcal{R}, \mathcal{H})$ and assume that (15) holds. Then

- for any given $N \geq 1$, (13) holds;

- the minimum number of modules for which (12) holds is given by $N=2$;

- for a planar configuration, i.e. $h_{i}=0$ for $i \in 1,2, \ldots N$, the minimum number of modules for which (12) holds is given by $N=3$.

Proof: The first item follows by contradiction showing that, if (13) is false, then necessarily Assumption 1 cannot hold true. Define $G\left(r_{i}, h_{i}\right):=\left[G_{f}^{T}, G_{\tau}^{T}\left(r_{i}, h_{i}\right)\right]^{T}$, denote by $G_{V T}\left(r_{i}, h_{i}\right)$ the last four rows of $G\left(r_{i}, h_{i}\right)$ and let $\bar{R}_{\psi_{i}}:=\operatorname{diag}\left(R_{\psi_{i}}, R_{\psi_{i}}\right)$. Since $\operatorname{rank}\left(R_{\psi_{i}}\right)=3$, we have $\operatorname{rank}\left(B_{V T}\right)<4$ if and only $\operatorname{rank}\left(G_{V T}\left(r_{i}, h_{i}\right)\right)<4$ for all $i \in\{1,2, \ldots N\}$. From the definition of $G_{f}$ and $G_{\tau}\left(r_{i}, h_{i}\right)$ given in subsection II-B, this implies that $h_{i}=d$ for all $i \in\{1,2, \ldots N\}$. However this fact contradicts Assumption 1 and, in turn, proves the claim in the first item.

To prove the second item, observe that, if $N=1$, then necessarily $r_{1}=h_{1}=0$ and then $\operatorname{rank}\left(B_{F A}\right)=$ $\operatorname{rank}(G(0,0))=4$. For $N=2$ let us consider the choice

$$
r_{1}:=r_{2}:=0, \quad h_{1}:=-h_{2}:=\bar{h}
$$

which corresponds to a system in which the two modules are connected on top of each others (see Figure 2(b)). Observe that (15) is satisfied for any choice of $\psi_{i} \in \mathbb{R}$, $i \in\{1,2\}$. Finally, assume, without loss of generality, to have $\psi_{1}=\psi_{2}=0$. The matrix $B_{F A}$ is then given by $B_{F A}=[G(0, \bar{h}), G(0,-\bar{h})]$. Simple computations show that $\operatorname{rank}(B)=6$ for all $\bar{h} \neq 0$. 
To prove the third item consider first a planar modular configuration with $N=2$. By construction, to satisfy (15), we have (see also Figure 1)

$$
r_{1}:=r_{2}:=\bar{r}, \quad h_{1}:=h_{2}:=0, \quad \psi_{2}:=\psi_{1}+\pi .
$$

Pick, without loss of generality, $\psi_{1}=0$. Then $B_{F A}=$ $\left[G(\bar{r}, 0), \bar{R}_{\pi} G(\bar{r}, 0)\right]$ whose rank is 5 for all $\bar{r}>0$. Finally, consider, for $N=3$, the following modular configuration which satisfies (15) (see Figure 2(a))

$$
\begin{gathered}
r_{1}:=r_{2}:=r_{3}:=\bar{r}, \quad h_{1}:=h_{2}:=h_{3}:=0 \\
\psi_{2}:=\psi_{1}-(2 / 3) \pi, \quad \psi_{3}:=\psi_{1}+(2 / 3) \pi
\end{gathered}
$$

Observe that the above planar modular configuration forms an equilateral triangle in which the length of each side is given by $\sqrt{3} \bar{r}$. By fixing $\psi_{1}=0$, we obtain

$$
B_{F A}=\left[G(\bar{r}, 0), \bar{R}_{-(2 / 3) \pi} G(\bar{r}, 0), \bar{R}_{(2 / 3) \pi} G(\bar{r}, 0)\right] .
$$

In this case, simple computations show that, for $\bar{r}>$ $0, \operatorname{rank}\left(B_{F A}\right)=6$, and then also the third item of the proposition holds true.

In the case in which additional payload is added to the modular system, the center of gravity of the overall modular vehicle may not coincide with the centroid of the formation as given in Assumption 1. The feasibility of the geometric properties (12)-(13) may be affected by the presence of a payload modifying the vertical position of the center of gravity of the overall formation, such as in the case in which the modular system is required to transport a heavy object. This kind of payload is denoted here as balanced since it does not affect the lateral and longitudinal position of the center of mass of the vehicle.

Assumption 2 (balanced payload): the body $z$-axis of the reference frame $F_{b_{m}}$ intersects the centroid formed by all the centers of mass of each single module, namely

$$
\sum_{i=1}^{N} R_{\psi_{i}} \ell_{i}^{b_{i}}=[0,0, \bar{z}]^{T}
$$

for some $\bar{z}$ in $\mathbb{R}$.

The effect of balanced payload in term of the geometric properties (12)-(13) is shown in the following proposition.

Proposition 2 Consider a modular system $\mathcal{M}=$ $(N, \Psi, \mathcal{R}, \mathcal{H})$ and assume that (20) holds. Then

- the minimum number of modules for which either (13) or (12) hold true for any $\bar{z} \in \mathbb{R}$ is given by $N=2$;

- for a planar configuration, i.e. $h_{i}=h_{j}$ for all $i \neq j$, $i, j \in 1,2, \ldots N$, the minimum number of modules for which either (13) or (12) hold true for any $\bar{z} \in \mathbb{R}$ is given by $N=3$.

Proof: Consider the definitions of $G\left(r_{i}, h_{i}\right)$, $G_{V T}\left(r_{i}, h_{i}\right)$ and $\bar{R}_{\psi_{i}}$ introduced in the proof of Proposition 1. Observe first of all that for $N=1$ by taking $r_{1}=0$ and $h_{1}=d$, assumption (20) is satisfied with $\bar{z}=d$ and
$\operatorname{rank}\left(G_{V T}(0, d)\right)=2$. Then pick the two-modules system defined in (16). From (5) if $h_{1}=d$ and $r_{1}=r_{2}=0$ then necessarily $h_{2} \neq d$. Assumption (20) is satisfied with $\bar{z}=h_{1}+h_{2}$ and the matrix $B_{F A}=\left[G\left(0, h_{1}\right), G\left(0, h_{2}\right)\right]$ has rank equal to 6 . This proves the first item.

To prove the second item, consider first the planar twomodules system defined by (17). If $h_{1}=h_{2}=d$ due to additional balanced payload (in fact (20) holds with $\bar{z}=2 d$ ) then $B_{V T}$ has rank equal to 3 . Finally, consider planar three-modules system defined in (18). In the worst case in which $h_{1}=h_{2}=h_{3}=d$, namely (20) holds with $\bar{z}=3 d$. Then, following the proof of Proposition 2, matrix $B_{F A}=$ $\left[G(\bar{r}, d), \bar{R}_{-(2 / 3) \pi} G(\bar{r}, d), \bar{R}_{(2 / 3) \pi} G(\bar{r}, d)\right]$ can be shown to have rank equal to 6 .

From the above result, it turns out that the presence of balanced payload may directly affect the minimum number of modules required to guarantee controllability of the system using the vectored-thrust paradigm. In fact the payload may reduce the distance between the resultant center of mass of the vehicle and the center of pressures of the aerodynamic surfaces and accordingly reduce the attitude control authority of the vehicle. Suitably designed modular systems are then shown to better tolerate variations in the position of the center of mass with respect to the single ducted-fan module.

\section{HOVERING IN THE PRESENCE OF DISTURBANCES}

In this section we show how the control allocation problems FA-CCAP and VT-CCAP can be employed to characterize the performances of the system in achieving interaction operations. In particular, with an eye on system (9), consider the problem of maintaining a stable hovering flight in the presence of external force and torque disturbances $f_{d}$ and $\tau_{d}$. In the hovering configuration the vehicle maintains a constant position $p=p^{\star}$ and a constant orientation such that $R e_{3}=$ $e_{3}$, namely the vertical body axis is perfectly aligned with the gravity force vector. This configuration is an equilibrium point if and only if it is possible to allocate a force vector $f_{c}^{\star}=f_{\text {hov }}\left(f_{d}\right)$ and a torque vector $\tau^{\star}=\tau_{c}^{\star}=\tau_{\text {hov }}\left(\tau_{d}\right)$ with

$$
f_{\text {hov }}\left(f_{d}\right):=-M_{m} g e_{3}-R^{T} f_{d}, \tau_{h o v}\left(\tau_{d}\right):=-\tau_{d} .
$$

Accordingly, if (12) holds for the given configuration, a necessary and sufficient condition for FA-CCAP to be feasible considering a desired wrench vector given by (21) is simply given by

$$
f_{c}^{\star} \in \Phi_{f} \text { and } \tau_{c}^{\star} \in \Phi_{\tau} .
$$

In the case in which only (13) holds, condition (22) is only necessary. Rather, a necessary and sufficient condition is to have, for some $\bar{w}^{\prime}$ such that $B_{\tau}(\Psi, \mathcal{R}, \mathcal{H}) \bar{w}^{\prime}=\tau_{c}^{\star}$,

$$
f_{c}^{\star} \in \Phi_{f}\left(\tau_{c}^{\star}\right)
$$

having defined

$$
\left\{f \in \Phi_{f} \mid f=B_{f} \bar{w}\left(\tau_{c}^{\star}\right), \begin{array}{l}
\Phi_{f}\left(\tau_{c}^{\star}\right):= \\
\left.\bar{w}\left(\tau_{c}^{\star}\right) \in \bar{w}^{\prime} \oplus \operatorname{ker}\left(B_{\tau}(\Psi, \mathcal{R}, \mathcal{H})\right)\right\} .
\end{array}\right.
$$




\section{A. Attainable Forces and Torques for a Physical Prototype}

In this section we compute the sets $\Phi_{f}$ and $\Phi_{\tau}$ by considering, for a single module, a physical ducted-fan prototype with $M=1.5 \mathrm{Kg}, \bar{\alpha}=0.4 \mathrm{rad}, \bar{T}=1.2$, $k_{L}=0.204 \mathrm{rad}^{-1}$ and $k_{L}^{\prime}=0.082 \mathrm{mrad}^{-1}$. In particular the parameters are obtained from the configuration described in [12] near the hovering condition. Figures 3(a)-3(b) and 4(a)-4(b) show the region of attainable forces and torques respectively for the single module and for the 2-modules prototype depicted in Figure 2(b) with $\bar{h}=0.7 \mathrm{~m}$. Observe that this 2-modules prototype preserves the same geometry of the original single module system, however, being fully actuated, any force and torque in $\Phi_{f} \times \Phi_{\tau}$ can be actually obtained. For the cases of planar modules with $N=2$ and $N=3$ (respectively Figure 1 and 2(a)), we consider the choice of $\bar{r}=0.7 \mathrm{~m}$ and we obtain the results shown in Figures 5(a)- 6(b). With respect to the previous cases observe that forces and torques polytopes are not symmetric.

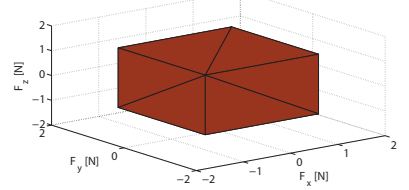

(a) Forces

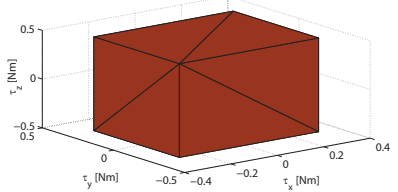

(b) Torques.
Fig. 3. The sets $\Phi_{f}$ and $\Phi_{\tau}$ for a single module prototype.

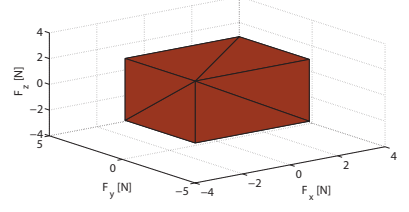

(a) Forces

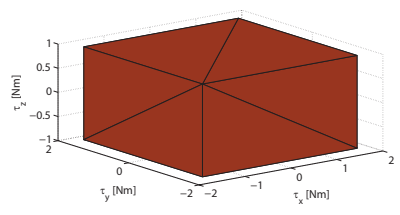

(b) Torques.
Fig. 4. The sets $\Phi_{f}$ and $\Phi_{\tau}$ for the two-module prototype depicted in Figure 2(b).

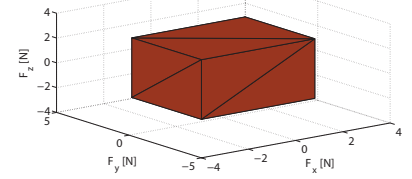

(a) Forces

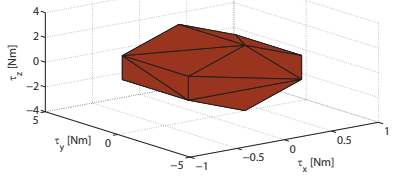

(b) Torques.
Fig. 5. The sets $\Phi_{f}$ and $\Phi_{\tau}$ for a the planar 2-modules prototype depicted in Figure 1.

\section{CONCLUSION}

In this work we have considered a class of aerial robots obtained by rigidly interconnecting a number of ducted-fan aerial vehicles. The dynamical properties of the system have been derived by solving suitable control allocation problems. The dependence of the obtained solutions on the geometrical properties of the modular system has been precisely pointed out showing how certain designs achieve better properties

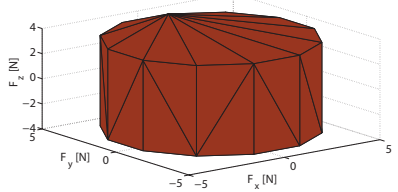

(a) Forces

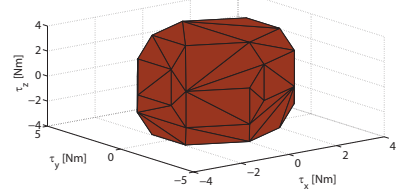

(b) Torques.
Fig. 6. The sets $\Phi_{f}$ and $\Phi_{\tau}$ for a the planar 3-modules prototype depicted in Figure 2(a).

in term of external disturbance rejection and robustness to payload variations.

\section{REFERENCES}

[1] Airobots project website. http://www.airobots.eu.

[2] A. Albers, S. Trautmann, T. Howard, T.A. Nguyen, M. Frietsch, and C. Sauter. Semi-autonomous flying robot for physical interaction with environment. In Proceedings of the IEEE Conference on Robotics Automation and Mechatronics (RAM), pages 441-446, Singapore, 2010.

[3] K.A. Bordignon. Constrained Control Allocation for Systems with Redundant Control Effectors. PhD. Thesis, Virginia Polytechnic Institute and State University, 1996.

[4] S. Bouabdallah and R. Siegwart. Advances in Unmanned Aerial Vehicles, chapter Chapter 6: Design and Control of a Miniature Quadrotor, pages 171-210. Springer Press, Feb. 2007.

[5] A. Casavola and E. Garone. Adaptive fault tolerant actuator allocation for overactuated plants. In Proceedings of the 2007 American Control Conference, New York City, USA, 2007.

[6] O. Härkegård. Backstepping and Control Allocation with Applications to Flight Control. PhD. Thesis, Department of Electrical Engineering, Linkping University, 2003.

[7] M.D. Hua, T. Hamel, P. Morin, and C. Samson. A control approach for thrust-propelled underactuated vehicles and its applications to vtol drones. IEEE Transsactions on Automatic Control, 54(8):1837-1853, 2009.

[8] A. Ko, O.J. Ohanian, and P. Gelhausen. Ducted fan uav modeling and simulation in preliminary design. In Proceedings of the AIAA Modeling and Simulation Technologies Conference and Exhibit, South Carolina, USA, 2007.

[9] L. Lipera, J.D. Colbourne, M.B. Tischler, M.H Mansur, M.C Rotkowitz, and $\mathrm{P}$ Patagui. The micro craft istar micro-air vehicle: Control system design and testing. Proc. of the 57th Annual Forum of the American Helicopter Society, 2001.

[10] L. Marconi, R. Naldi, and L. Gentili. Modeling and control of a flying robot interacting with the environment. accepted to Automatica, 2011.

[11] D. Mellinger, M. Shomin, N. Michael, and V. Kumar. Cooperative grasping and transport using multiple quadrotors. In Proceedings of the International Symposium on Distributed Autonomous Systems, Lausanne, Switzerland, 2010.

[12] R. Naldi, L. Gentili, L. Marconi, and A. Sala. Design and experimental validation of a nonlinear control law for a ducted-fan miniature aerial vehicle. Control Engineering Practice, 18(7):747-760, 2010.

[13] R. Oung, F. Bourgault, M. Donovan, and R. DAndrea. The distributed flight array. In Proceedings of the 2010 IEEE International Conference on Robotics and Automation, Anchorage, Alaska, USA, 2010.

[14] B. Siciliano and O. Khatib, editors. Springer Handbook of Robotics. Springer, 2008.

[15] R.F. Stengel. Flight Dynamics. Princeton University Press, 2004.

[16] B. Thwaites. Incompressible Aerodynamics. Oxford University Press, 1960.

[17] M.W. Zhao and C. Bill. Aerodynamic design and analysis of a vtol ducted-fan uav. In Proceeding of the 26th AIAA Applied Aerodynamics Conference, Honolulu, Hawaii, USA, 2008. 